\title{
Establishment virtual maintenance environment based on VIRTOOLS to effectively enhance the sense of immersion of teaching equipment
}

\author{
Fushuan $\mathrm{Wu}^{1, \text { a }}$, Zhenguo Liu ${ }^{1, \text { a }}$, Jianren Wang ${ }^{1, \text { a }}$, Yuzhuo Zhao ${ }^{2, \mathrm{~b}}$ \\ ${ }^{1}$ Wuhan institute of machinery, Wuhan, 430075, China \\ ${ }^{2}$ Wuhan University, Wuhan, 430075, China \\ a1286697@qq.com
}

Keywords: virtual simulation; immersion; VIRTOOLS; equipment maintenance

\begin{abstract}
There is the lack of immersion in the maintenance training system based on the virtual reality technology, which leads to the learners can't understand and grasp the learning content accurately and comprehensively, influencing the effect of teaching..In this paper, from the visual, hearing, touch, direction,expression and language aspects to discuss how to improve the immersion of virtual maintenance training system. This paper presents research teaching based on VIRTOOLS of equipment maintenance virtual training systems, which will bring the equipment maintenance teaching new vitality. The system combine the virtual reality technology and equipment maintenance training teaching,effectively solve the number of quality venues, training efficiency and other prominent contradictions in teaching mounting equipment maintenance training equipment.
\end{abstract}

\section{Introduction}

Virtual reality technology is the integration of artificial intelligence technology, multimedia technology, computer graphics technology and computer network technology and developed into a new computer human-computer interaction technology, users can experience the three-dimensional virtual environment computer-generated, and naturally make a real-time interaction with virtual environment from visual, auditory and even tactile and taste,obtaining the similar operating experience with real-world.With the rapid development of advanced simulation technology, in the army's teaching and training, equipped with virtual maintenance training system based on the virtual reality technology is going to be "generation with soft and hard, with virtual to real mixing" of a new mode of teaching and training, it is widely used in the training and teaching, playing an important role in improving the teaching and training quality.Enhancing the immersion design of equipment virtual training system, can promote the students' flow experience, making it become closer to equipment maintenance training environment, play the subjective initiative of students' cognition, better close to the practical requirements.

Virtual reality interactive software Virtools 5.0 has the characteristics of strong interaction, good portability, and low operating environment requirements. Combined with practical training equipment, this paper presents the Equipment Maintenance virtual training systems based on Virtools 5.0.On the basis of virtual reality, distributed simulation and other high technology, combines the virtual reality technology and basic aspects in equipment maintenance training,ensuring the good person machine interaction, making the operator trainers immersion feel, effectively enhancing the sense of immersion of virtual equipment teaching.

\section{Enhance the immersion of equipment virtual maintenance training system}

The so-called immersion refers to a realistic felling that allows users to generate their own seem to have exposure to the virtual environment, and can perceive and manipulate various objects in the virtual world, but also to actively participate in various events.

Immersion produced mainly from the human perception system and the behavior system. Perception system consists of human vision, hearing, touch, smell and taste and so on; behavior 
system consists of human posture, orientation, motion, movement, expression and language.Mainly in the visual, auditory, tactile three aspects of perceptual system, and direction, expression, language three aspects of behavior system in the of successful virtual.

\section{To enhance the visual immersion}

Equipment Virtual Maintenance Training System development is to establish realistic model by collecting a variety of data of real equipment, and use three-dimensional graphics construct virtual scene,enhancing the visual immersion should focus on the model establishment of a scene, the sense of space design, light-efficient design and texture design.

1)Set up model(modeling)

Modeling is the key to produce virtual maintenance training system.Geometry modeling usually use 3dmax, Maya and other three-dimensional modeling software. Models include high-precision models and low accuracy of the model. The advantage of high-precision model is richly detailed, edges smooth, but the drawback is its too many faces, large files, and slower running. The disadvantage of low accuracy of the model is its low precision, but it can use mapping to achieve realistic effects, with a small file size, fast running advantage. The main components can take high-precision model modeling, while sub-components by low accuracy modeling method, so that can guarantee the results, at the same time, can reduce the complexity of the scene in real-time play-dyed, optimize the number of model faces, improve running speed .

2)Sense of space design

Sense of space generated mainly from the scene geometry perspective and aerial perspective relationship between objects. To ensure the geometric relationships between objects perspective, in the virtual scene, camera parameter settings should be used to meet the vision and perspective of the human eye when viewing an object, making the objects in the virtual scene in line near bigger the far smaller, near low the far high, near coarse the far fine, near wide the far narrow of the law.

3)Light Efficiency Design

Light effects can express different environment and atmosphere, light-efficient design is another important factor affecting the virtual maintenance training system visual immersion. 3Dmax, Maya and other software can simulate the effect of different light source light on the object, and its rendering engine can real-time present the object surface's color and brightness,resulting in a true natural effect.

4)Texture design

Good design can fully reflect the realistic texture detail of components, can enhance the visual immersion of virtual maintenance training system effectively.In the geometric modeling foundation of basic body parts on the use of mapping, can be given a realistic texture and delicate texture of the object, without increasing the complexity of the model can show more details. Surveying techniques for generating components through panoramic three-dimensional effect, the high-resolution camera on the basis of the photos you can handle light color rendering more realistic texture.

\section{Auditory immersion upgrade}

In equipment virtual maintenance training system, auditory immersion mainly through the virtual sound information.Voice messages mainly through tone, intonation, even stop, stress, rhythm softly and organic changes and melody, charm and strength to create a different state of mind and mood to improve auditory immersion.Ethereal voice can create a sense of technology, echo can create a sense of open space, deep voice can create a mysterious atmosphere, cheerful music gives a sense of movement, soothing sounds can create a sense of relief. In addition, changes in the size of the sound should be able to reflect changes in body position, the position of the object from far to near, the sound should be small to large, and vice versa.

\section{Tactile immersion upgrade}

In general, the surface texture, the soft and hard, the surface roughness can be provided by the surface information produced by the skin, and shape of the object, characterized in elasticity can be provided through the movement of the hand and arm resulting perceptual information. As such, in equipment virtual maintenance training system production, while using the hand or other body parts 
operate the virtual parts, the information should be used between the surface and the body and virtual components generated virtual components to enhance the tactile immersion.

\section{Language system immersion upgrade}

Language is mankind's most natural form of communication, if the operator can freely use the language and the environment structured by the virtual reality system to communicate, it will definitely raise the sense of immersion. Microphone is the hardware base of language system, the key technology is the voice recognition technology and voice synthesis technology. By recognizing the participants' language, the user can issue commands to the virtual reality system, or chat with others in a virtual environment.

\section{Expression system immersion upgrade}

In virtual reality, expression system is the use of expression gestures, facial expressions, posture, etc. to convey certain information. Input devices of gesture recognition system is based on the identification data is divided into gloves and based on visual (image) of two sign language recognition system. The general process is: first start using a camera continuously shooting after the motion picture department, and then take each finger contour recognition approach shot on the turn with a boundary feature recognition method to distinguish a small, continuous gestures.

\section{Building of virtual equipment maintenance environment based on VIRTOOLS}

\section{Build equipment model}

Three-dimensional model is the foundation of the entire equipment maintenance simulation system, which directly affects the truth of virtual maintenance. This article is equipped with a mechanical product model, choose the professional mechanical CAD software to model three-dimensional graphics. When modeling, integrated use of the sense of space design, light design and texture design methods can effectively enhance immersion. Before modeling, we should conduct a preliminary analysis, divide the complex mechanical products into several parts and then make three-dimensional modeling of the simple various components. After good model and successfully imported into $3 \mathrm{dsmax} 2009$, the job is giving to the model materials and textures, increase realistic feel of models and reduce unnecessary polygon,increase the display refresh rate. Three-dimensional model of a certain type of equipment shown in Figure 1.

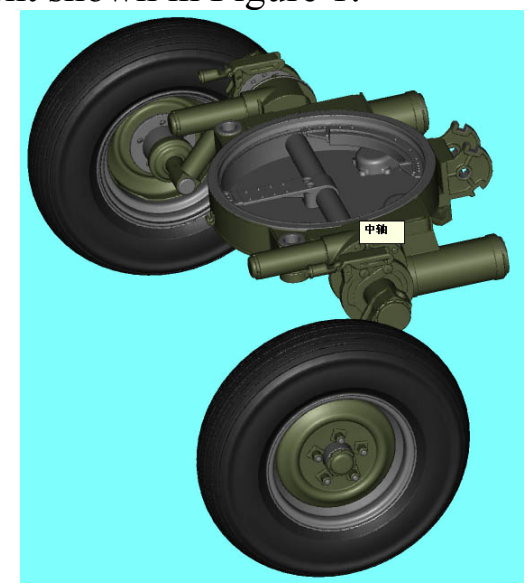

Fig1 . 3D model of a certain type of equipment

\section{Build model display system}

Virtools has produced an immersion virtual environment, it makes its participants generate a variety of sensory information such as vision, hearing, touch, etc, giving the participants an immersion feel, is a new development, with a new meaning kinds of human-computer interaction system. Virtools primarily through a well-designed graphical user interface, use modular elements of behavior modules compose interactions scripting language. This allows the user to quickly familiar with the various functions, ranging from simple deformation to mechanical function. In accordance with the mounting dimensions and testing maintenance environment, the use of $3 \mathrm{dmax} 2009$ software 
to build equipment parts digital three-dimensional structural model of high resolution. Based on the model, light, textures, animation and video cameras added processing, using Virtools 5.0 graphics engine driving digital model, while adding properties such as strength, reminders etc. auxiliary maintenance factor, making the whole scene lifelike, high maneuverability, to meet a variety of service level maintenance training.

Equip each part by drawing software CAD modeling, and then built a good model into 3 dsmax2009, and then exported by 3 dsmax2009 into Virtools 5.0 to run.

\section{Message implementation of Equipment Maintenance Simulation}

Control between each equipment maintenance simulation display interfaces rely on Virtools 5.0 message handling mechanisms implementation. System messages and custom message map with the specified action are shown in Figure 2 . Interactive modules using these behaviors can be flexible control flow passing messages between different objects, and in accordance with the requirements of the user habits and flexible control the virtual characters.

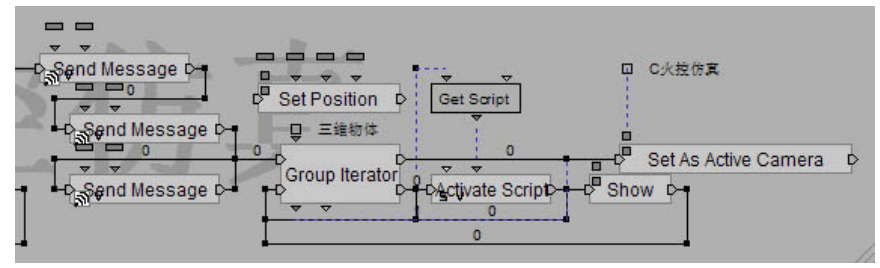

Fig 2.Message passing

Virtools provides behavioral interaction module Send Message, Send Message To Message, Wait Message, Broadcast Message,supporting message transmit between different messaging control flow and different objects, including system messages and custom messages, and use Check For Message, Get Message Data, Switch On Message, etc. BB to receive messages and data. Send Message need to specify the content of the message and the recipient. Send Message To Message send the message to a specified target group, while Wait Message and Check For Message check the specified message, Get Message Data can read the message accompanying data, Switch On Message will respond differently depending on the message. These two acts by interaction module can achieve a dynamic link between the different control flows and different objects, making the whole system organic, orderly fashion.

\section{Virtual equipment}

Virtual equipment is based on the shape characteristics and precision characteristics of the product design, using a group of part models realistically simulate a three-dimensional product assembly process, and allows the user to interactively control the three-dimensional simulation of the real assembly process of the product, to test the product can be assembled. This definition focuses on the physical simulation of product assembly process, reflects an analytic process. The key technology of virtual equipment is the parts and assemblies distance discriminant collision, they can be achieved by using dedicated collision VSL BBS and design provided by Virtools 5.0. Figure 3 is a type of virtual assembly equipment.

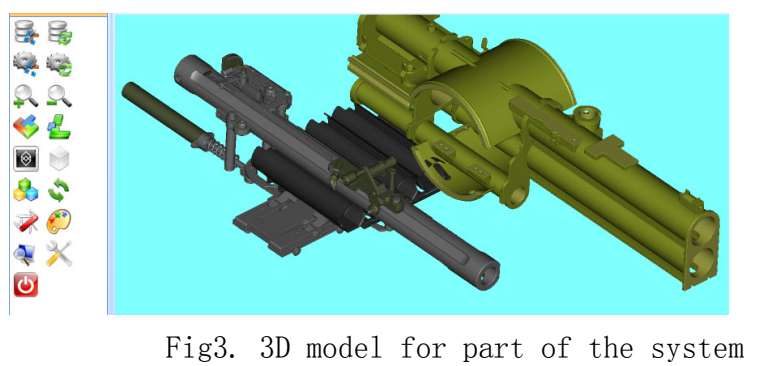

\section{Conclusion}

This paper,mainly from the visual, hearing, touch,expression and language of behavior system of perception system discussing to enhance the virtual maintenance training system immersion.Virtools is a new interactive system between person and machine. Virtools technology with its characteristics 
of powerful interactive design capabilities, visualization and easy operation, so that in the development of virtual experiment in equipment maintenance, equipment maintenance greatly enhanced interactivity and immersion, improved the efficiency and quality of virtual construction of vocational educational equipment maintenance the reality. Virtual reality platform based on Virtools software provides a useful supplement for practical equipment teaching.Currently, it has been designed and developed the initial realization of the basic functions, with good effect. As a teaching visual simulation technology, it provides virtual equipment environment with immersion and interaction, stimulates the students for professional experimental initiative and dynamism.

\section{References}

[1]Zhenguo Liu, Fu Jiang, Bin Shen, Tao Wang,Xin Yu,Wensheng Huang. Virtual Laboratory for Equipment Repair based on VIRTOOLS [J].Applied Mechanics and Materials,ISSN:1662-7482.

[2] Hu Yong, Naikun Wei, Dianliang Wu, Xiumin Fan,Assembly design collaborative virtual environments based systems [J]. Computer Engineering, 2010.6.

[3]D. Tian and N. Georganas. A coverage-Preserving Node Scheduling Scheme for Large Wireless Sensor Networks. 2002, 9. 